\title{
La CACG, organe de gestion de l'eau : un instrument technique et un lieu de concertation
}

\author{
Henri Tardieu \\ Directeur Général - CACG
}

\section{Introduction}

La sécheresse de l'été 1991 confirme une nouvelle fois la faiblesse des ressources en eau naturelle disponibles en Midi-Pyrénées. A nouveau, moins de $20 \mathrm{~m}^{3} / \mathrm{s}$ pour la Garonne à Toulouse, c'est très insuffisant pour garantir la salubrité du fleuve qui exige un DMA (débit minimum admissible) de $55 \mathrm{~m}^{3} / \mathrm{s}$.

Depuis 1988, tous les partenaires se sont mobilisés en Adour-Garonne pour lancer le Programme Décennal de Ressources en eau (PDRE), avec l'ambition de restaurer partout un débit d'étiage correct tout en réalisant un aménagement qui respecte l'environnement.

Il faut créer de nouvelles ressources en eau. Pour convaincre, il faut aussi mettre en place une nouvelle méthode de gestion des eaux qui garantisse l'efficacité de ces aménagements par rapport au développement des prélèvements.

La « méthode CACG » est décrite ici à propos de l'exemple du "Système Neste " : $1300 \mathrm{~km}$ de rivières réalimentées par plusieurs ouvrages stockant et distribuant en été 100 millions de $\mathrm{m}^{3}$. Cette méthode pose en principe la priorité au débit d'étiage des rivières, le développement des prélèvements n'étant autorisé que dans la limite des nouvelles ressources créées. Elle marie une haute technologie pour le contrôle des débits en temps réel, et une pratique de la concertation fondée sur les contrats individuels et collectifs.

\section{Un peu de géographie et d'histoire}

\subsection{Quelques idées sur le climat de Gascogne. La complé- mentarité avec les Pyrénées}

La seule eau naturelle disponible en Gascogne, c'est la pluie qui tombe et ruisselle sur les collines argileuses imperméables. Aucun apport extérieur ne provient naturellement des Pyrénées à cause de l'accident géologique du plateau de Lannemezan et par ailleurs aucune nappe importante, facilement accessible, ne permet de gérer un "capital eau " dont on pourrait user en année de crise.

L'eau qui ruisselle quitte la Gascogne au bout de quelques heures ou quelques jours; on doit la stocker pour en avoir la disponibilité pendant l'été. Or, pour une pluviométrie annuelle de 600 à $900 \mathrm{~mm}$, le ruissellement mesuré s'établit pour une année de sécheresse banale (fréquence 1 année sur 5) de 75 à $100 \mathrm{~mm}$ (rappelons que $1 \mathrm{~mm}$ correspond à $1000 \mathrm{~m}^{3}$ pour $1 \mathrm{~km}^{2}$ de bassin versant). Certaines années de crise grave (1967 et 1990), on a constaté des ruissellements inférieurs à $20 \mathrm{~mm}$.

A proximité immédiate de la Gascogne, se trouvent les Pyrénées pour lesquelles le volume d'eau ruisselé est 10 fois supérieur ( 700 à $900 \mathrm{~mm}$ ) pour une année de sécheresse de fréquence quinquennale.

Il y a donc une évidente complémentarité climatique entre les Pyrénées et la Gascogne, d'autant qu'une partie des écoulements des Pyrénées peut se prolonger sur une ou deux décades du mois de juillet pendant la plus forte période de besoins en eau en Gascogne.

Cette complémentarité Gascogne-Pyrénées présente un autre intérêt, plus complexe à analyser. Il s'agit des variances comparées entre le ruissellement pyrénéen et les ruissellements en Gascogne. En effet, nous savons que les pluviométries annuelles en Gascogne sont très variables.

Mais la maîtrise de l'eau fait appel plus au ruissellement qu'à la pluie elle-même et la caractéristique de la Gascogne est que la variabilité du ruissellement naturel d'une année sur l'autre est encore plus marquée que celle des pluies (coefficient de variation $48,8 \%$ ).

\begin{tabular}{|l|c|c|}
\hline & $\begin{array}{c}\text { Coefficient de } \\
\text { variation } \\
\text { écart type/ } \\
\text { moyenne }\end{array}$ & $\begin{array}{c}\text { Plus faible valeur, } \\
\text { en \%, } \\
\text { de la moyenne } \\
\text { sur 25 ans }\end{array}$ \\
\hline $\begin{array}{l}\text { Pluie annuelle } \\
\text { en Gascogne }\end{array}$ & $18,4 \%$ & $70 \%$ \\
\hline $\begin{array}{l}\text { Ruissellement } \\
\text { annuel en } \\
\text { Gascogne }\end{array}$ & $48,8 \%$ & $12 \%$ \\
\hline $\begin{array}{l}\text { Ruissellement } \\
\text { Pyrénéen (Neste) }\end{array}$ & $16,8 \%$ & $78 \%$ \\
\hline
\end{tabular}


A contrario, les ruissellements pyrénéens, caractéristiques d'un régime pluvio-nival, sont beaucoup moins aléatoires puisque on constate un coefficient de variation de $16,8 \%$.

Ainsi, la Gascogne se caractérise par une ressource naturelle en eau faible et très aléatoire, mais elle a la chance d'être proche des Pyrénées dont la ressource en eau est beaucoup plus riche, beaucoup plus stable et décalée vers le printemps et le début de l'été.

\subsection{Brève histoire d'un aménagement}

Dès le début du $\mathrm{XIX}^{\mathrm{e}}$ siècle, les ingénieurs ont pensé que la proximité des Pyrénées est une chance pour la Gascogne. L'aménagement hydraulique s'est concrétisé en 1863 par la mise en service du Canal de la Neste, long de $28 \mathrm{~km}$, qui permettait de transiter $7 \mathrm{~m}^{3} / \mathrm{s}$ vers toutes les rivières de Gascogne, de la Save à la Baïse.

A l'origine, l'aménagement visait à satisfaire plusieurs catégories d'utilisateurs : les éleveurs pour l'abreuvement du bétail, les agriculteurs pour l'irrigation, les scieurs ou meuniers pour l'utilisation de la force motrice et bien sûr les transporteurs pour ce projet un peu fou de navigation sur les rivières. L'alimentation en eau potable des populations est progressivement devenue l'une des utilités essentielles de cet aménagement au fur et à mesure du développement de l'action des Communes en ce domaine.

Même si les Pyrénées étaient une chance pour la Gascogne, il est très vite apparu nécessaire de conforter l'alimentation du canal de la Neste par des barrages en montagne; finalement ce sont 48 millions de mètres cubes qui sont réservés à l'alimentation de la Gascogne au moyen du Canal de la Neste dont la capacité a simultanément été portée à $14 \mathrm{~m}^{3} / \mathrm{s}$ en 1955 .

A partir de la création de la CACG, en 1960, démarre à la fois une nouvelle politique de ressource en eau et une augmentation progressive des prélèvements à usage d'irrigation.

Ainsi, sont créés des réservoirs de stockage en tête de rivière de Gascogne destinés à compléter l'alimentation de ces rivières pendant la période d'étiage. Ces stockages ont le mérite de pouvoir être remplis par les eaux de printemps de la Neste grâce au Canal. Ainsi, la variabilité de remplissage est celle - faible - des écoulements pyrénéens. On a vu, ces dernières années, l'importance de cette garantie de remplissage pour passer les hivers extrêmement secs que nous avons dû subir.

Avec la mise en service du barrage "Gimone", les réservoirs complémentaires ( 54 millions de $\mathrm{m}^{3}$ ) ainsi créés portent à 100 millions de volumes stockés disponibles pour alimenter la Gascogne.

Cependant, les apports printaniers du bassin versant de la Neste ont une limite qui est maintenant atteinte et une autre solution doit être mise en œuvre pour l'avenir : c'est l'aménagement de "CHARLAS » qui permettra de valoriser les eaux hivernales de la Garonne elle-même, comme cela a d'ailleurs été inscrit dans les textes par nos anciens (Décret de 1963).

\begin{tabular}{|c|c|}
\hline \multicolumn{2}{|c|}{$\begin{array}{l}\text { Quelques dates marquantes } \\
\text { dans l'évolution du système Neste }\end{array}$} \\
\hline 1863 & Mise en service du Canal de la Neste \\
\hline $1886-1909$ & $\begin{array}{l}\text { Décrets de répartition du débit de salu- } \\
\text { brité véhiculé par le Canal de la Neste }\end{array}$ \\
\hline $1950-1955$ & $\begin{array}{l}\text { Revêtement du Canal de la Neste - Créa- } \\
\text { tion de I'Institution Interdépartementale } \\
\text { pour I'Aménagement Hydraulique des } \\
\text { Coteaux de Gascogne }\end{array}$ \\
\hline 1960 & $\begin{array}{l}\text { Création de la Compagnie d'Aménage- } \\
\text { ment des Coteaux de Gascogne (CACG) } \\
\text { et attribution d'une dotation sur le Canal } \\
\text { de la Neste }\end{array}$ \\
\hline 1963 & $\begin{array}{l}\text { Décret de répartition des eaux Neste et } \\
\text { Garonne }\end{array}$ \\
\hline $1965-1967$ & $\begin{array}{l}\text { Réalimentation de l'Osse. } \\
\text { Barrage de Miélan et transfert Baïse- } \\
\text { Osse }\end{array}$ \\
\hline 1970 & $\begin{array}{l}\text { Réfection de la prise du Canal de la Neste } \\
\text { à Sarrancolin }\end{array}$ \\
\hline 1976 & $\begin{array}{l}\text { Mise en service du barrage de l'Astarac } \\
\left(10 \mathrm{hm}^{3}\right) \text { (Arrats) }\end{array}$ \\
\hline 1987 & $\begin{array}{l}\text { Mise en service du barrage de } \\
\text { Puydarrieux }\left(14,5 \mathrm{hm}^{3}\right) \text { (Baîse) }\end{array}$ \\
\hline 1990 & $\begin{array}{l}\text { Concession du Canal de la Neste à la } \\
\text { CACG et début du programme de restau- } \\
\text { ration et de modernisation }\end{array}$ \\
\hline 1991 & $\begin{array}{l}\text { Mise en service du barrage Gimone } \\
\left(25 \mathrm{hm}^{3}\right) \text { " Lunax" (Gimone-Save) }\end{array}$ \\
\hline
\end{tabular}

\section{Des moyens techniques modernes au service d'une gestion de l'eau au compte-gouttes}

L'eau de la Gascogne est pour l'essentiel artificielle bien qu'elle utilise les vecteurs naturels que sont les $1300 \mathrm{~km}$ de rivières.

Pour l'ingénieur, c'est une responsabilité importante que d'utiliser les moyens techniques les plus modernes au service d'une gestion économe de cette ressource en eau chère et difficile à mobiliser.

Sans s'étendre sur ces aspects techniques de la gestion des eaux qui a déjà fait l'objet de nombreux articles, rappelons quelques-uns des éléments mis en place depuis moins de 10 ans à ce sujet.

\subsection{La gestion automatique des barrages (gestion tactique)}

Envoyer dans la rivière l'eau tout juste nécessaire à la satisfaction des besoins est la première condition de cette gestion des eaux. Il convient en effet de faire coïncider les lâchures opérées par les barrages, ou à partir des canaux, 


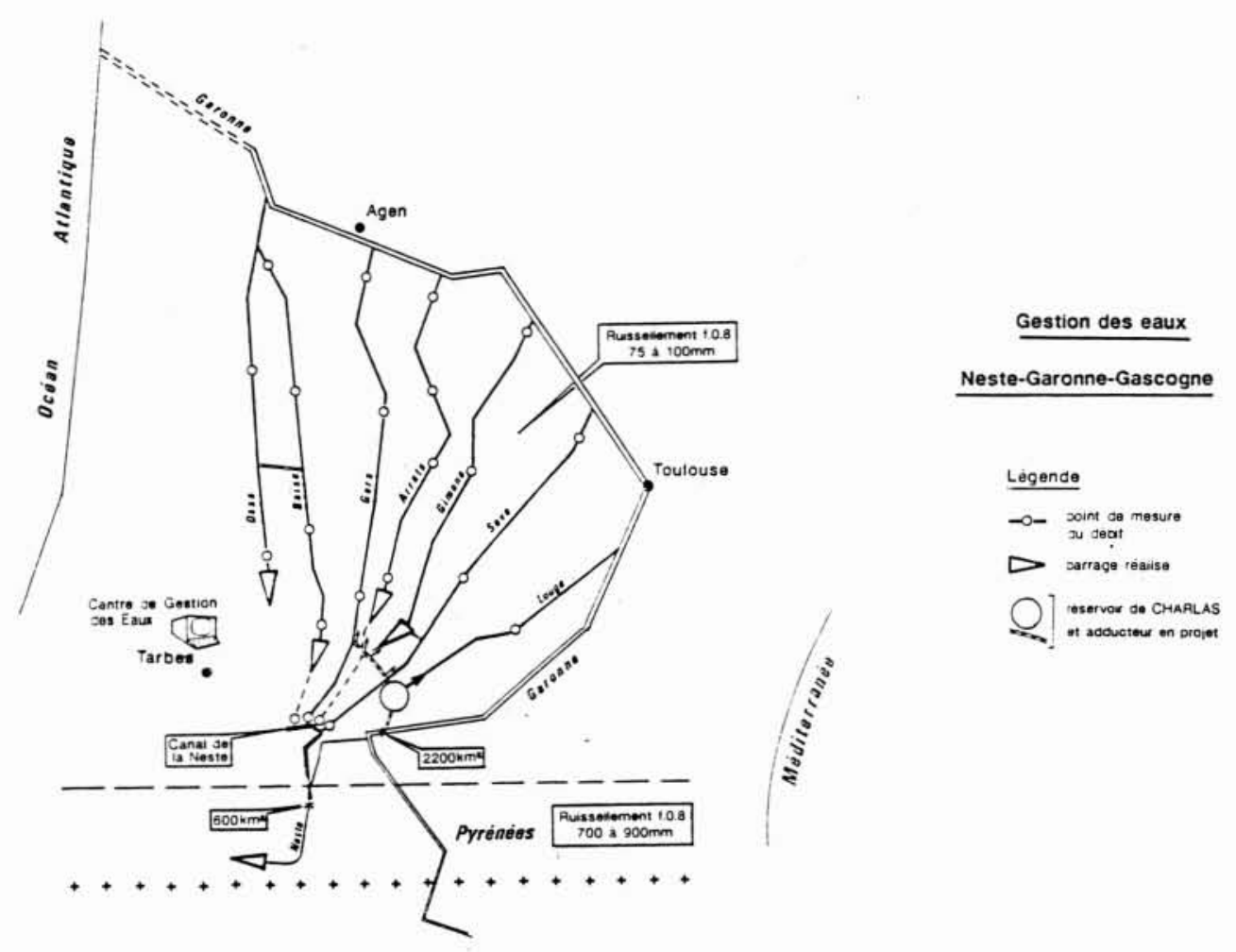

avec des besoins en eau qui sont eux-mêmes variables en fonction de la situation climatique du moment. Cette adéquation est d'autant plus difficile que le temps de transport entre les réserves amont et le dernier irrigant à l'aval est de plusieurs jours.

Ainsi un logiciel de gestion automatique a été développé consistant en une commande du barrage, assurée par un micro-ordinateur, compte tenu de mesures de débit en temps réel et de paramètres régulièrement mis à jour par l'opérateur. Le cycle d'interrogation et d'ajustement de la commande est de 3 heures.

La mise en place depuis 6 ans de cette commande automatique a permis d'économiser, pour les barrages qui en sont équipés à l'heure actuelle, de l'ordre de $20 \%$ du volume stocké.

Le système complet de gestion automatique est actuellement mis en place sur 3 barrages (Astarac, Puydarrieux et Gimone) ; un système moins sophistiqué de télégestion est en place sur 7 autres barrages. Les vannes de distribution du Canal de la Neste seront progressivement équipées par un système analogue.

Soulignons que l'ensemble des logiciels est implanté sur plusieurs micro-ordinateurs compatibles, raccordés aux stations de mesures par le réseau France Télécom. Ceci permet à la fois une grande robustesse des équipements, mais également une bonne transparence de l'information, accessible en tout point par minitel.

Ce dernier aspect est essentiel dans la «méthode CACG ", car la transparence fonde aussi la confiance dans les relations avec les différents acteurs.

\subsection{La gestion stratégique}

C'est celle qui, au pas de temps de la semaine, va permettre d'adapter les paramètres de la gestion tactique pour faire face aux événements risquant de se produire pendant la période de gestion des eaux ( $1^{\text {er }}$ juin au 28 février).

En effet, analyser la situation hydrologique et climatique, esquisser des prévisions de consommation et de ressource, apprécier les risques futurs et leurs enjeux économiques, arbitrer l'ensemble pour définir une stratégie optimum de réponse à la sécheresse, tout cela ne s'improvise pas.

Il a donc été mis au point un modèle d'optimisation des stratégies en temps réel, destiné à prendre de meilleures décisions d'utilisation des ouvrages et de restrictions éventuelles des usages. Ce modèle travaillant au pas de temps de la semaine à l'horizon de quelques mois, est fondé sur une double approche :

- définition de "bonnes stratégies" fondées sur une analyse du passé permettant de situer en fréquence la stratégie actuelle avec quantification de l'éventail des possibles. La réduction des incertitudes est accrue par les quelques modèles de prévisions possibles (prévision des étiages, des consommations d'eau d'irrigation);

- optimisation d'une fonction économique permettant, dans le lot des «bonnes stratégies ", de retenir la meilleure par les méthodes de programmation dynamique.

Utilisé à l'essai dès 1990 et opérationnel en 1991, ce modèle de gestion stratégique a donné de bons résultats. 
Il a notamment aidé à préserver les réserves nécessaires à la salubrité des rivières pendant l'automne, malgré la pression exercée par la demande d'irrigation.

\section{Concertation entre les acteurs et gestion des rela- tions contractuelles}

Outre l'outil technique, permettant de gérer la ressource en eau et de donner la réponse exacte et nécessaire aux besoins exprimés, il est mis en place un outil de concertation où s'établissent les règles du jeu entre les usagers de l'eau.

Il s'agit de la Commission Neste, émanation du Conseil d'administration de la CACG, donc représentant les cinq départements et les deux régions concernés au niveau des élus, des administrations, de la profession agricole, ouverte aux représentants directs des irrigants et aux autres partenaires (Agence de l'eau, EDF, notamment).

Quelles sont donc les principales règles de gestion?

\subsection{Le contrat individuel}

L'usage de l'eau des rivières réalimentées par le système Neste est régi par un contrat de droit privé entre les usagers et la CACG. Pour les irrigants, ce contrat prend le nom de Convention de Restitution. Il concerne également les communes et les industriels utilisateurs d'eau brute.

C'est en effet par la relation contractuelle qu'est régi l'essentiel des questions de gestion des eaux, ne plaçant la réglementation préfectorale qu'en recours ultime en cas de crise.

Le contrat fixe le débit et le volume d'eau maximum prélevés par l'usager signataire en fonction desquels sont injectés le débit et les volumes nécessaires pour répondre aux besoins. Il fixe également le tarif de ce service et les moyens de contrôle acceptés (notamment la mise en place de compteurs de l'eau prélevée). Cette relation contractuelle est confortée par une autorisation administrative de prélèvement qui lui est attachée. Ceci permet de recourir aux moyens classiques de la police des eaux placée sous la responsabilité du Préfet, en cas de non-observance des règles par l'usager.

Mais le contrat vise également les obligations de la CACG et les risques encourus par elle en cas de défaillance du système. Il ne s'agit donc pas seulement d'une autorisation de prélèvement, mais bien d'une garantie de fourniture d'eau assortie de certaines limites.

\subsection{Le contrat collectif: partage de l'eau avant l'été}

Si les règles d'usage de l'eau sont fixées par le contrat individuel, la gestion collective de la ressource en eau s'impose notamment en cas de sécheresse. C'est ainsi qu'il est prévu de procéder à un partage des ressources disponibles entre les différents usages juste avant l'été.

Il s'agit d'abord, pour les techniciens, d'évaluer la ressource disponible en tenant compre, dans le cas du système Neste, des ressources naturelles prévisibles du fait des écoulements d'étiage de la Neste. Ensuite, le point très précis doit être fait des usages à cette date. Ceci suppose un fichier complet, actualisé en permanence, suivi en transparence avec les cinq DDAF chargées de la police des eaux.

L'expérience montre que ce fichier des usagers doit être simple si on veut qu'il soit complet et à jour : même si l'informatique peut théoriquement tout, les gestionnaires du fichier saturent très vite devant la multiplicité des informations dont on peut s'inventer le besoin à propos des problèmes d'eau.

Pour ce qui nous concerne, nous nous contentons de l'identification du préleveur et de son lieu de prélèvement, du débit et du volume qu'il est autorisé à prélever et du débit et du volume supplémentaires qu'il souhaiterait pouvoir utiliser. Cette dernière information est essentielle car le système Neste étant saturé depuis 1989, il convient aussi de gérer la file d'attente des demandes.

Le partage de l'eau avant l'été entre usages se résume en fait à un partage essentiel visant à réserver, aux dépens de l'irrigation, le volume nécessaire à la salubrité des rivières et à la fabrication de l'eau potable pour l'automne. C'est ainsi que, malgré l'extrême tension qui prévalait avant l'été 1990, 15 millions de mètres cubes ont pu être réservés dans les barrages pour garantir l'alimentation des rivières de Gascogne, en vue d'un étiage qui, certaines années, se prolonge jusqu'en février.

Le partage des volumes réservés à l'irrigation conduit à la définition d'un quota en volume affecté à chaque irrigant. Ce quota est à la fois pour chacun une garantie de la ressource et une incitation à l'économie. Il vise à responsabiliser chaque irrigant face à la gestion commune de l'eau.

Ce quota est fixé en principe à $4000 \mathrm{~m}^{3}$ pour chaque litre par seconde souscrit. En 1990, il a été fixé le 30 mai - à la suite de la réunion de concertation - à $2700 \mathrm{~m}^{3} / \mathrm{l} / \mathrm{s}$, et ajusté le 6 juillet à $3000 \mathrm{~m}^{3} / 1 / \mathrm{s}$ en fonction de l'évolution réelle des débits naturels. En 1991, le quota a pu être fixé normalement à $4000 \mathrm{~m}^{3} / \mathrm{l} / \mathrm{s}$.

Ce principe des quotas n'a été accepté par les irrigants que dans la mesure où, d'une part, il se situe à un niveau suffisamment élevé en année normale pour n'être pas une contrainte lourde, et que, d'autre part, une diminution des quotas en cas d'insuffisance des ressources conduit à une implication financière de la Compagnie.

Soulignons que l'introduction de la notion de quota d'eau est assortie d'une réflexion agro-économique sur la gestion des assolements : l'eau ne doit plus être considérée comme un intrant banal à prix défini et à disponibilité illimitée pour lequel la consommation optimum est celle dont le coût annule la productivité marginale. On doit considérer l'eau disponible en débit et en volume comme une contrainte physique au même titre que les surfaces cultivables. Ainsi, le partage de l'eau permet à chacun de connaître la limite du «capital » dont il peut disposer une année donnée. 


\subsection{Contrôle et sanctions : la question du droit de l'eau}

Un système collectif, comportant près de 3000 préleveurs, ne peut pas fonctionner sans contrôle, même si l'accent est mis sur la concertation et le consensus.

Le moyen technique essentiel de ce contrôle a été l'installation obligatoire d'un compteur de volume sur chaque prélèvement en rivière, permettant à la fois de vérifier le débit prélevé et de suivre l'utilisation du quota d'eau.

La plupart des stations de pompage pour l'eau potable sont équipées de ce dispositif. En revanche, il a été nécessaire d'installer près de 1800 compteurs d'eau sur les installations de pompage individuelles. Ce compteur est muni d'une étiquette informative rappelant les conditions du contrat (localisation du prélèvement, débit, identification du préleveur, etc...).

Pour exercer le contrôle du débit prélevé et du volume consommé en cours de saison, les cinq préfectures concernées ont commissionné une trentaine d'agents de la CACG pour exercer un contrôle officiel au titre de la police des eaux.

Le Code Rural dans son état actuel permet l'application de sanctions : néanmoins, les contentieux ouverts à la suite des infractions constatées en 1989 et 1990 ne sont pas complètement réglés, en partie à cause d'un manque de clarté du droit en matière de prélèvement dans les cours d'eau non domaniaux.

Il est urgent que la nouvelle loi sur l'eau lève définitivement les ambiguïtés en confirmant la possibilité, pour l'Administration, de limiter quantitativement les prélèvements d'eau. Ceci confirmerait la validité de la méthode développée ici.

\section{Oui aux contraintes à condition de poursuivre l'aménagement}

La méthode de gestion ainsi décrite concerne les crises mais aussi, pour le système Neste, la gestion en année normale. Elle impose beaucoup de contraintes aux utilisateurs, notamment un arrêt total du développement de l'irrigation dès que les ressources en eau sont insuffisantes.

Il est clair que ceci n'est possible que si en même temps des perspectives de déblocage sont envisagées grâce à un développement des ressources en eau. En effet, dans le contexte économique de l'agriculture du Sud-Ouest, la décision d'arrêter la délivrance d'autorisations de pompage nouvelles est perçue comme un blocage du développement de l'agriculture. Une telle décision ne peut tenir que si des assurances sont données sur la mise en œuvre de barrages nouveaux, sinon - soyons réalistes - au diable les contraintes, au diable le civisme, la gestion des crises devient impossible. En Gascogne, la poursuite de l'aménagement, c'est la réalisation du projet de Charlas. Ce projet aura également pour mérite d'étendre la nouvelle méthode de gestion des eaux à l'ensemble de la vallée de la Garonne qui sera également réalimentée par Charlas.

Il convient de ne pas rompre le fil de l'Histoire, même s'il est bien clair que le contexte a changé, notamment au niveau de l'opinion publique. La qualité de la méthode de gestion des eaux mise en œuvre en Gascogne devrait précisément permettre de convaincre l'opinion que ces projets de barrage sont l'ultime réponse, après que tout ait été mis en œuvre en matière d'économie et de gestion rationnelle de l'eau. 